\title{
The arrowhead-Pell-random-type sequences
}

\author{
Özgür Erdağ ${ }^{1}$, Anthony G. Shannon ${ }^{2}$ and Ömür Deveci ${ }^{1}$ \\ ${ }^{1}$ Department of Mathematics, Faculty of Science and Letters \\ Kafkas University 36100, Turkey \\ e-mails: ozgur_erdag@hotmail.com, odeveci36@hotmail.com \\ ${ }^{2}$ Fellow, Warrane College, The University of New South Wales \\ Kensington, 2033, Australia \\ e-mail: tshannon38@gmail.com
}

Received: 17 November 2016

Accepted: 31 January 2018

\begin{abstract}
In this paper, we define the arrowhead-Pell-random-type sequence and then we obtain the generating function and the generating matrix of the sequence. Also, we derive the permanental, determinantal, combinatorial and exponential representations and the sums of the arrowhead-Pell-random-type numbers using the generating function and the generating matrix of the sequence.
\end{abstract}

Keywords: The arrowhead-Pell numbers, Sequence, Matrix.

2010 Mathematics Subject Classification: 11B50, 11C20, 20 D60.

\section{Introduction}

In [13], Kilıc and Tasci defined $k$ sequences of the generalized order- $k$ Pell numbers as shown:

$$
P_{n}^{i}=2 P_{n-1}^{i}+P_{n-2}^{i}+\cdots+P_{n-k}^{i}
$$

for $n>0$ and $1 \leq i \leq k$, with initial conditions

$$
P_{n}^{i}=\left\{\begin{array}{ll}
1 & \text { if } n=1-i, \\
0 & \text { otherwise, }
\end{array} \quad 1-k \leq n \leq 0,\right.
$$

where $P_{n}^{i}$ is the $n$-th term of the $i$-th sequence.

It is clear that the characteristic polynomial of the generalized order- $k$ Pell sequence is as follows:

$$
P(x)=x^{k}-2 x^{k-1}-x^{k-2}-\cdots-1 .
$$


In [1], Aküzüm et al. defined the arrowhead-Pell sequence for $n \geq 1$ as follows:

$$
a_{k+1}(n+k+1)=a_{k+1}(n+k)-2 a_{k+1}(n+k-1)-a_{k+1}(n+k-2)-\cdots-a_{k+1}(n)
$$

with integer constants $a_{k+1}(1)=\cdots=a_{k+1}(k)=0$ and $a_{k+1}(k+1)=1$, where $k \geq 2$.

Shannon and Horadam [17] also developed arrowhead curves in the context of recursive sequences.

Hofstadter's integer sequences defined [10] by

$$
h_{n}=h_{n-h_{n-1}}+h_{n-h_{n-2}}
$$

where $h_{1}=h_{2}=1$.

The random Fibonacci sequences defined [6] by the random recurrence $x_{1}=1, x_{2}=2$ and for $n>2, x_{n}= \pm x_{n-1} \pm x_{n-2}$, where each \pm sign is independent and either + or - with probability $1 / 2$.

Atanassov et al. [2] have, to some extent, systematized aspects of these sequences through pulsated sequences.

Suppose that the $(n+k)$ th term of a sequence is defined recursively by a linear combination of the preceding $k$ terms:

$$
a_{n+k}=c_{0} a_{n}+c_{1} a_{n+1}+\cdots+c_{k-1} a_{n+k-1}
$$

where $c_{0}, c_{1}, \ldots, c_{k-1}$ are real constants. In [11], Kalman derived a number of closed-form formulas for the generalized sequence by the companion matrix method as follows:

$$
A=\left[a_{i, j}\right]_{k \times k}=\left[\begin{array}{cccccc}
0 & 1 & 0 & \cdots & 0 & 0 \\
0 & 0 & 1 & \cdots & 0 & 0 \\
0 & 0 & 0 & \ddots & 0 & 0 \\
\vdots & \vdots & \vdots & & \vdots & \vdots \\
0 & 0 & 0 & \cdots & 0 & 1 \\
c_{0} & c_{1} & c_{2} & & c_{k-2} & c_{k-1}
\end{array}\right] .
$$

Then by an inductive argument he obtained that

$$
A^{n}\left[\begin{array}{c}
a_{0} \\
a_{1} \\
\vdots \\
a_{k-1}
\end{array}\right]=\left[\begin{array}{c}
a_{n} \\
a_{n+1} \\
\vdots \\
a_{n+k-1}
\end{array}\right]
$$

for $n \geqslant 0$.

Number theoretic properties such as these obtained from homogeneous linear recurrence relations relevant to this paper have been studied by many authors $[4,7,8,9,12,16,18,19$, $20,21,22,23]$. In this paper, we define a new sequence which is called the arrowhead-Pellrandom-type sequence. Then we give relationships among the arrowhead-Pell-random-type numbers and the permanents and the determinants of certain matrices which are produced by using the 
generating matrix of the arrowhead-Pell-random-type sequence. Also, we obtain the combinatorial representations, the exponential representation and the sums of the arrowhead-Pell-randomtype numbers by the aid of the generating function and the generating matrix of the arrowheadPell-random-type sequence.

\section{The arrowhead-Pell-random-type sequence}

We now define the arrowhead-Pell-random-type sequence by the following recurrence relations for $n \geq u$

$$
\begin{aligned}
a_{k+1}^{u}(n+k+1) & =a_{k+1}^{u}(n+k-u)-2 a_{k+1}^{u}(n+k-u-1) \\
& -a_{k+1}^{u}(n+k-u-2)-\cdots-a_{k+1}^{u}(n-u)
\end{aligned}
$$

with initial conditions $a_{k+1}^{u}(0)=\cdots=a_{k+1}^{u}(u+k-1)=0$ and $a_{k+1}^{u}(u+k)=1$, where $1 \leq u \leq k+1$ and $k \geq 2$.

By (2.1), we can write a generating matrix for the arrowhead-Pell-random-type sequence as follows:

$$
A^{k, u}=\left[\begin{array}{cccccccc}
0 & \cdots & 0 & 1 & -2 & -1 & \cdots & -1 \\
1 & 0 & 0 & 0 & 0 & 0 & \cdots & 0 \\
0 & 1 & 0 & 0 & 0 & 0 & \cdots & 0 \\
0 & 0 & 1 & 0 & 0 & 0 & \cdots & 0 \\
0 & 0 & 0 & 1 & 0 & 0 & \cdots & 0 \\
\vdots & \vdots & \vdots & \ddots & \ddots & \ddots & & \vdots \\
0 & 0 & 0 & \cdots & 0 & 1 & 0 & 0 \\
0 & 0 & 0 & 0 & \cdots & 0 & 1 & 0
\end{array}\right]_{(u+k+1) \times(u+k+1)}
$$

The companion matrix $A^{k, u}$ is called the arrowhead-Pell-random-type matrix. It is clear that

$$
\left(A^{k, u}\right)^{\alpha}\left[\begin{array}{c}
a_{k+1}^{u}(u+k) \\
a_{k+1}^{u}(u+k-1) \\
\vdots \\
a_{k+1}^{u}(0)
\end{array}\right]=\left[\begin{array}{c}
a_{k+1}^{u}(\alpha+u+k) \\
a_{k+1}^{u}(\alpha+u+k-1) \\
\vdots \\
a_{k+1}^{u}(\alpha)
\end{array}\right]
$$

for $\alpha \geq u$. Let $a_{k+1}^{u}(\alpha)$ be denoted by $a_{k+1}^{u, \alpha}$. By induction on $\alpha$, we derive that 


$$
\left(A^{k, u}\right)^{\alpha}=\left[\begin{array}{cccccc}
a_{k+1}^{u, \alpha+u+k} & a_{k+1}^{u, \alpha+u+k+1} & \ldots & a_{k+1}^{u, \alpha+2 u+k} & a_{k+1}^{u, \alpha+2 u+k+1}-a_{k+1}^{u, \alpha+u+k} & \\
a_{k+1}^{u, \alpha+u+k-1} & a_{k+1}^{u, \alpha+u+k} & \cdots & a_{k+2 u+k-1}^{u, \alpha+1} & a_{k+1}^{u, \alpha+2 u+k}-a_{k+1}^{u, \alpha+u+k-1} & \\
\vdots & \vdots & & \vdots & \vdots \\
a_{k+1}^{u, \alpha+u} & a_{k+1}^{u, \alpha+u+1} & \cdots & a_{k+1}^{u, \alpha+2 u} & a_{k+1}^{u, \alpha+2 u+1}-a_{k+1}^{u, \alpha+u} & \left(M^{u, k}\right)^{\alpha} \\
a_{k+1}^{u, \alpha+u-1} & a_{k+1}^{u, \alpha+u} & \cdots & a_{k+1}^{u, \alpha+2 u-1} & a_{k+1}^{u, \alpha+2 u}-a_{k+1}^{u, \alpha+u-1} & \\
\vdots & \vdots & \ddots & \vdots & \vdots \\
a_{k+1}^{u, \alpha} & a_{k+1}^{u, \alpha+1} & \cdots & a_{k+1}^{u, \alpha+u} & a_{k+1}^{u, \alpha+u+1}-a_{k+1}^{u, \alpha} &
\end{array}\right]
$$

for $k \geq 2$. Where $\left(M^{k, u}\right)^{\alpha}$ is a $(u+k+1) \times(k-2)$ matrix as follows:

$$
\begin{gathered}
\left(M^{k, u}\right)^{\alpha} \\
=\left[\begin{array}{cc}
-a_{k+1}^{u, \alpha+u+1}-a_{k+1}^{u, \alpha+u+2}-\ldots-a_{k+1}^{u, \alpha+u+k-1}-a_{k+1}^{u, \alpha+u+2}-a_{k+1}^{u, \alpha+u+3}-\ldots-a_{k+1}^{u, \alpha+u+k-1} & \ldots \\
-a_{k+1}^{u, \alpha+u}-a_{k+1}^{u, \alpha+u+1}-\ldots-a_{k+1}^{u, \alpha+u+k-2}-a_{k+1}^{u, \alpha+u+1}-a_{k+1}^{u, \alpha+u+2}-a_{k+1}^{u, \alpha+u+k-2} & \ldots \\
\vdots & \left.M^{* k, u}\right)^{\alpha} \\
-a_{k+1}^{u, \alpha-k+1}-a_{k+1}^{u, \alpha-k+2}-\ldots-a_{k+1}^{u, \alpha-1}-a_{k+1}^{u, \alpha+k+2}-a_{k+1}^{u, \alpha-k+3}-\ldots-a_{k+1}^{u, \alpha-1} & \ldots
\end{array}\right]
\end{gathered}
$$

such that

$$
\left(M^{* k, u}\right)^{\alpha}=\left[\begin{array}{c}
-a_{k+1}^{u, \alpha+u+k-1} \\
-a_{k+1}^{u, \alpha+u+k-2} \\
\vdots \\
-a_{k+1}^{u, \alpha-1}
\end{array}\right]_{(u+k+1) \times 1}
$$

from which it is clear that $\operatorname{det}\left(A^{k, u}\right)^{\alpha}=(-1)^{\alpha(u+k+1)}$.

Now we consider the permanental representations of the arrowhead-Pell-random-type sequence.

Definition 2.1. A $u \times v$ real matrix $M=\left[m_{i, j}\right]$ is called a contractible matrix in the $k^{\text {th }}$ column (resp. row.) if the $k^{\text {th }}$ column (resp. row.) contains exactly two non-zero entries.

Suppose that $x_{1}, x_{2}, \ldots, x_{u}$ are row vectors of the matrix $M$. If $M$ is contractible in the $k^{\text {th }}$ column such that $m_{i, k} \neq 0, m_{j, k} \neq 0$ and $i \neq j$, then the $(u-1) \times(v-1)$ matrix $M_{i j: k}$ obtained from $M$ by replacing the $i^{\text {th }}$ row with $m_{i, k} x_{j}+m_{j, k} x_{i}$ and deleting the $j^{\text {th }}$ row. The $k^{\text {th }}$ column is called the contraction in the $k^{\text {th }}$ column relative to the $i^{\text {th }}$ row and the $j^{\text {th }}$ row.

In [3], Brualdi and Gibson obtained that $\operatorname{per}(M)=\operatorname{per}(N)$ if $M$ is a real matrix of order $\alpha>1$ and $N$ is a contraction of $M$.

Let $m \geqslant u+k+1$ be a positive integer and suppose that $H^{k, u}(m)=\left[h_{i, j}^{m, k, u}\right]$ is the $m \times m$ super-diagonal matrix, defined by: 


$$
h_{i, j}^{m, k, u}=\left\{\begin{array}{cc} 
& \text { if } i=r \text { and } j=r+u \text { for } 1 \leq r \leq m-u-k \\
& \text { and } \\
& i=r+1 \text { and } j=r \text { for } 1 \leq r \leq m-1, \\
& \text { if } i=r \text { and } j=r+u+2 \text { for } 1 \leq r \leq m-u-k, \\
-1 & i=r \text { and } j=r+u+3 \text { for } 1 \leq r \leq m-u-k, \\
& \vdots \\
-2 & i=r \text { and } j=r+u+k \text { for } 1 \leq r \leq m-u-k, \\
0 & \text { if } i=r \text { and } j=r+u \text { for } 1 \leq r \leq m-u-1, \\
& \text { otherwise. }
\end{array}\right.
$$

That is,

$$
\begin{aligned}
& (u+1) \text { th } \quad(u+k+1) \text { th } \\
& H^{k, u}(m)=\left[\begin{array}{ccccccccccccc}
0 & \ldots & 0 & 1 & -2 & -1 & \ldots & -1 & 0 & 0 & 0 & \ldots & 0 \\
1 & 0 & \ldots & 0 & 1 & -2 & -1 & \ldots & -1 & 0 & 0 & \ldots & 0 \\
0 & 1 & 0 & \ldots & 0 & 1 & -2 & -1 & \ldots & -1 & 0 & \ldots & 0 \\
\vdots & \ddots & \ddots & \ddots & & \ddots & \ddots & \ddots & \ddots & & \ddots & \ddots & \vdots \\
0 & \ldots & 0 & 1 & 0 & \ldots & 0 & 1 & -2 & -1 & \ldots & -1 & 0 \\
0 & 0 & \ldots & 0 & 1 & 0 & \ldots & 0 & 1 & -2 & -1 & \cdots & -1 \\
\vdots & \vdots & \ddots & & \ddots & \ddots & \ddots & & \ddots & \ddots & \ddots & \ddots & \vdots \\
0 & 0 & 0 & 0 & \ldots & 0 & 1 & 0 & \ldots & 0 & 1 & -2 & -1 \\
0 & 0 & 0 & 0 & 0 & \ldots & 0 & 1 & 0 & \ldots & 0 & 1 & -2 \\
0 & 0 & 0 & 0 & 0 & 0 & \ldots & 0 & 1 & 0 & \ldots & 0 & 1 \\
0 & 0 & 0 & 0 & 0 & 0 & 0 & \ldots & 0 & 1 & 0 & \ldots & 0 \\
\vdots & \vdots & \vdots & \vdots & \vdots & \vdots & \vdots & \ddots & & \ddots & \ddots & \ddots & \vdots \\
0 & 0 & 0 & 0 & 0 & 0 & 0 & 0 & 0 & \ldots & 0 & 1 & 0
\end{array}\right]
\end{aligned}
$$

Then we have the following Theorem.

Theorem 2.1. For $m \geqslant u+k+1$ and $k \geq 2$,

$$
\operatorname{per} H^{k, u}(m)=a_{k+1}^{u}(m+u+k) .
$$

Proof. Let the equation hold for $m \geqslant u+k+1$, then we show that the equation holds for $m+1$. If we expand the $\operatorname{per} H^{k, u}(m)$ by the Laplace expansion of permanent with respect to the first row, then we obtain

$$
\begin{aligned}
\operatorname{per} H^{k, u}(m+1) & =\operatorname{per} H^{k, u}(m-u)-2 \operatorname{per} H^{k, u}(m-u-1)-\operatorname{per} H^{k, u}(m-u-2) \\
& -\cdots-\operatorname{per} H^{k, u}(m-u-k) .
\end{aligned}
$$

Since

$$
\begin{aligned}
\operatorname{per} H^{k, u}(m-u) & =a_{k+1}^{u}(m+k), \\
\operatorname{per} H^{k, u}(m-u-1) & =a_{k+1}^{u}(m+k-1),
\end{aligned}
$$




$$
\operatorname{per} H^{k, u}(m-u-2)=a_{k+1}^{u}(m+k-2), \ldots, \operatorname{per} H^{k, u}(m-u-k)=a_{k+1}^{u}(m),
$$

we easily obtain that $\operatorname{per}^{k, u}(m+1)=a_{k+1}^{u}(m+u+k+1)$.

So the proof is complete. by

Let $m>u+k+1$ such that $k \geq 2$ and let $L^{k, u}(m)=\left[l_{i, j}^{m, k, u}\right]$ be the $m \times m$ matrix, defined

$$
l_{i, j}^{m, k, u}=\left\{\begin{array}{cc} 
& \text { if } i=r \text { and } j=r+u \text { for } 1 \leq r \leq m-u-k \\
& \text { and } \\
& i=r+1 \text { and } j=r \text { for } 1 \leq r \leq m-1, \\
& \text { if } i=r \text { and } j=r+u+2 \text { for } 1 \leq r \leq m-u-k, \\
-1 & i=r \text { and } j=r+u+3 \text { for } 1 \leq r \leq m-u-k, \\
& \\
& i=r \text { and } j=r+u+k \text { for } 1 \leq r \leq m-u-k, \\
-2 & \text { if } i=r \text { and } j=r+u \text { for } 1 \leq r \leq m-u-k, \\
0 & \text { otherwise. }
\end{array}\right.
$$

That is,

$$
\begin{aligned}
& (u+1) \text { th } \quad(u+k+1) \text { th } \\
& L^{k, u}(m)=\left[\begin{array}{ccccccccccccc}
0 & \ldots & 0 & 1 & -2 & -1 & \ldots & -1 & 0 & 0 & 0 & \ldots & 0 \\
1 & 0 & \ldots & 0 & 1 & -2 & -1 & \ldots & -1 & 0 & 0 & \ldots & 0 \\
0 & 1 & 0 & \ldots & 0 & 1 & -2 & -1 & \ldots & -1 & 0 & \cdots & 0 \\
\vdots & \ddots & \ddots & \ddots & & \ddots & \ddots & \ddots & \ddots & & \ddots & \ddots & \vdots \\
0 & \ldots & 0 & 1 & 0 & \ldots & 0 & 1 & -2 & -1 & \ldots & -1 & 0 \\
0 & 0 & \cdots & 0 & 1 & 0 & \cdots & 0 & 1 & -2 & -1 & \cdots & -1 \\
0 & 0 & 0 & \ldots & 0 & 1 & 0 & \cdots & 0 & 0 & 0 & 0 & 0 \\
\vdots & \vdots & \vdots & \ddots & & \ddots & \ddots & \ddots & \vdots & \vdots & \vdots & \vdots & \vdots \\
0 & 0 & 0 & 0 & 0 & \cdots & 0 & 1 & 0 & 0 & 0 & 0 & 0 \\
0 & 0 & 0 & 0 & 0 & 0 & \cdots & 0 & 1 & 0 & 0 & 0 & 0 \\
0 & 0 & 0 & 0 & 0 & 0 & 0 & \cdots & 0 & 1 & 0 & 0 & 0 \\
0 & 0 & 0 & 0 & 0 & 0 & 0 & 0 & \cdots & 0 & 1 & 0 & 0 \\
0 & 0 & 0 & 0 & 0 & 0 & 0 & 0 & 0 & \cdots & 0 & 1 & 0
\end{array}\right] \leftarrow(m-u-k) \text { th. }
\end{aligned}
$$

Assume that the $m \times m$ matrix $K^{k, u}(m)=\left[k_{i, j}^{m, k, u}\right]$ is defined by 


$$
K^{k, u}(m)=\left[\begin{array}{ccccc}
-1 & \cdots & -1 & 0 & 0 \\
1 & & & & \\
0 & & & \\
0 & & & L^{k, u}(m-1) \\
\vdots & & &
\end{array}\right]
$$

Then we can give more general results by using other permanental representations than the above.

Theorem 2.2. Let $a_{k+1}^{u}(m)$ be the mth the arrowhead-Pell-random-type number for $k \geq 2$. Then (i). For $m>u+k+1$,

$$
\operatorname{per} L^{k, u}(m)=-a_{k+1}^{u}(m-1)
$$

(ii). For $m>u+k+2$,

$$
\operatorname{per} K^{k, u}(m)=\sum_{i=1}^{m-2} a_{k+1}^{u}(i) .
$$

Proof. (i). Let the equation hold for $m>u+k+1$, then we show that the equation holds for $m+1$. If we expand the $\operatorname{per} L^{k, u}(m)$ by the Laplace expansion of permanent with respect to the first row, then we obtain

$$
\begin{aligned}
\operatorname{per} L^{k, u}(m+1) & =\operatorname{per} L^{k, u}(m-u)-2 \operatorname{per} L^{k, u}(m-u-1)-\operatorname{per} L^{k, u}(m-u-2) \\
& -\cdots-\operatorname{per} L^{k, u}(m-u-k) .
\end{aligned}
$$

Also, since

$$
\begin{gathered}
\operatorname{per} L^{k, u}(m-u)=-a_{k+1}^{u}(m-u-1), \\
\operatorname{per} L^{k, u}(m-u-1)=-a_{k+1}^{u}(m-u-2), \\
\operatorname{per} L^{k, u}(m-u-2)=-a_{k+1}^{u}(m-u-3), \ldots, \\
\operatorname{per} L^{k, u}(m-u-k)=-a_{k+1}^{u}(m-u-k-1),
\end{gathered}
$$

it is clear that

$$
\operatorname{per} L^{k, u}(m+1)=-a_{k+1}^{u}(m) .
$$

(ii). It is clear that expanding the per $K^{k, u}(m)$ by the Laplace expansion of permanent with respect to the first row, gives us

$$
\operatorname{per} K^{k, u}(m)=\operatorname{per} K^{k, u}(m-1)+\operatorname{per} L^{k, u}(m-1) .
$$

By induction on $m$, taking into consideration the result of Theorem 2.1 and part (i) in Theorem 2.2 , the conclusion is easily seen. 
Let the notation $A \circ K$ denotes the Hadamard product of $A$ and $K$. A matrix $A$ is called convertible if there is an $m \times m(1,-1)$-matrix $K$ such that per $A=\operatorname{det}(A \circ K)$.

Let $m>u+k+2$ and let $R$ be the $m \times m$ matrix, defined by

$$
R=\left[\begin{array}{cccccc}
1 & 1 & 1 & \cdots & 1 & 1 \\
-1 & 1 & 1 & \cdots & 1 & 1 \\
1 & -1 & 1 & \cdots & 1 & 1 \\
\vdots & \ddots & \ddots & \ddots & \ddots & \vdots \\
1 & \cdots & 1 & -1 & 1 & 1 \\
1 & \cdots & 1 & 1 & -1 & 1
\end{array}\right]
$$

It is easy to see that $\operatorname{per} H^{k, u}(m)=\operatorname{det}\left(H^{k, u}(m) \circ R\right), \operatorname{per} L^{k, u}(m)=\operatorname{det}\left(L^{k, u}(m) \circ R\right)$ and $\operatorname{per} K^{k, u}(m)=\operatorname{det}\left(K^{k, u}(m) \circ R\right)$ for $m>u+k+2$. Then we have the following useful results.

Corollary 2.3. For $m>u+k+2$,

$$
\begin{gathered}
\operatorname{det}\left(H^{k, u}(m) \circ R\right)=a_{k+1}^{u}(m+u+k), \\
\operatorname{det}\left(L^{k, u}(m) \circ R\right)=-a_{k+1}^{u}(m-1)
\end{gathered}
$$

and

$$
\operatorname{det}\left(K^{k, u}(m) \circ R\right)=\sum_{i=1}^{m-2} a_{k+1}^{u}(i)
$$

Let $C\left(c_{1}, c_{2}, \ldots, c_{v}\right)$ be a $v \times v$ companion matrix as follows:

$$
C\left(c_{1}, c_{2}, \ldots, c_{v}\right)=\left[\begin{array}{cccc}
c_{1} & c_{2} & \cdots & c_{v} \\
1 & 0 & \cdots & 0 \\
\vdots & \ddots & \vdots & \vdots \\
0 & \cdots & 1 & 0
\end{array}\right] .
$$

See $[14,15]$ for more information about the companion matrix.

Theorem 2.4 (Chen and Louck [5]). The $(i, j)$ entry $c_{i, j}^{(n)}\left(c_{1}, c_{2}, \ldots, c_{v}\right)$ in matrix $C^{n}\left(c_{1}, c_{2}, \ldots\right.$, $\left.c_{v}\right)$ is given by the following formula:

$$
c_{i, j}^{(n)}\left(c_{1}, c_{2}, \ldots, c_{v}\right)=\sum_{\left(t_{1}, t_{2}, \ldots, t_{v}\right)} \frac{t_{j}+t_{j+1}+\cdots+t_{v}}{t_{1}+t_{2}+\cdots+t_{v}} \times\left(\begin{array}{c}
t_{1}+\cdots+t_{v} \\
t_{1}, \ldots, t_{v}
\end{array}\right) c_{1}^{t_{1}} \cdots c_{v}^{t_{v}}
$$

where the summation is over nonnegative integers satisfying $t_{1}+2 t_{2}+\cdots+v t_{v}=n-i+j$, $\left(\begin{array}{c}t_{1}+\cdots+t_{v} \\ t_{1}, \ldots, t_{v}\end{array}\right)=\frac{\left(t_{1}+\cdots+t_{v}\right) !}{t_{1} ! \cdots t_{v} !}$ is a multinomial coefficient, and the coefficients in (2.2) are defined to be 1 if $n=i-j$.

Then we give the combinatorial representations for the arrowhead-Pell-random-type numbers. 
Corollary 2.5. Let $a_{k+1}^{u}(\alpha)$ be the $\alpha$ th the arrowhead-Pell-random-type number for $k \geq 2$. Then

$$
a_{k+1}^{u}(\alpha)=\sum_{\left(t_{1}, t_{2} \ldots, t_{u+k+1}\right)}\left(\begin{array}{c}
t_{1}+\cdots+t_{u+k+1} \\
t_{1}, \ldots, t_{u+k+1}
\end{array}\right)(-2)^{t_{u+2}}(-1)^{t_{u+3}+t_{u+4}+\cdots+t_{u+k+1}}
$$

where the summation is over nonnegative integers satisfying $t_{1}+2 t_{2}+\cdots+(u+k+1) t_{u+k+1}=$ $\alpha-u-k$.

(ii) $\quad a_{k+1}^{u}(\alpha)$

$=-\sum_{\left(t_{1}, t_{2} \ldots, t_{u+k+1}\right)} \frac{t_{u+k+1}}{t_{1}+t_{2}+\cdots+t_{u+k+1}} \times\left(\begin{array}{c}t_{1}+\cdots+t_{u+k+1} \\ t_{1}, \ldots, t_{u+k+1}\end{array}\right)(-2)^{t_{u+2}}(-1)^{t_{u+3}+t_{u+4}+\cdots+t_{u+k+1}}$

where the summation is over nonnegative integers satisfying $t_{1}+2 t_{2}+\cdots+(u+k+1) t_{u+k+1}=$ $\alpha+1$.

Proof. If we take $i=u+k+1, j=1, c_{1}=\cdots=c_{u}=0, c_{u+1}=1, c_{u+2}=-2, c_{u+3}=\cdots=$ $c_{u+k+1}=-1$ for the case $(i)$. and $i=u+k, j=u+k+1, c_{1}=\cdots=c_{u}=0, c_{u+1}=1$, $c_{u+2}=-2, c_{u+3}=\cdots=c_{u+k+1}=-1$ for the case $(i i)$. in Theorem 2.4, then the proof is immediately seen from $\left(M^{k, u}\right)^{\alpha}$.

It is easy to show that the generating function of the arrowhead-Pell-random-type sequence is as follows:

$$
g^{k, u}(x)=\frac{x^{u+k}}{1-x^{u+1}+2 x^{u+2}+x^{u+3}+\cdots+x^{u+k+1}},
$$

where $k \geq 2$.

Now we give an exponential representation for the arrowhead-Pell-random-type numbers by the aid of the generating function with the following Theorem.

Theorem 2.6. The arrowhead-Pell-random-type numbers have the following exponential representation:

$$
g^{k, u}(x)=x^{u+k} \exp \left(\sum_{i=1}^{\infty} \frac{\left(x^{u+1}\right)^{i}}{i}\left(1-x-\cdots x^{k}\right)^{i}\right)
$$

where $k \geq 2$.

Proof. Since

$$
\ln g^{k, u}(x)=\ln x^{u+k}-\ln \left(1-x^{u+1}+2 x^{u+2}+x^{u+3}+\cdots+x^{u+k+1}\right)
$$

and

$$
\begin{aligned}
-\ln \left(1-x^{u+1}+2 x^{u+2}+x^{u+3}+\cdots+x^{u+k+1}\right)= & -\left[-x^{u+1}\left(1-2 x-x^{2}-\cdots x^{k}\right)-\right. \\
& \frac{1}{2}\left(x^{u+1}\right)^{2}\left(1-2 x-x^{2}-\cdots x^{k}\right)^{2}-\cdots \\
& \left.-\frac{1}{n}\left(x^{u+1}\right)^{n}\left(1-2 x-x^{2}-\cdots x^{k}\right)^{n}-\cdots\right],
\end{aligned}
$$


it is clear that

$$
\ln g^{k, u}(x)-\ln x^{u+k}=\ln \frac{g^{k, u}(x)}{x^{u+k}}=\sum_{i=1}^{\infty} \frac{\left(x^{u+1}\right)^{i}}{i}\left(1-2 x-x^{2}-\cdots x^{k}\right)^{i} .
$$

Thus we have the conclusion.

Now we consider the sums of arrowhead-Pell-random-type numbers.

Let

$$
S_{\alpha}=\sum_{i=1}^{\alpha} a_{k+1}^{u}(i)
$$

for $\alpha>1$ and $k \geq 2$, and suppose that $E^{u, k}$ is the $(u+k+2) \times(u+k+2)$ matrix such that

$$
E^{k, u}=\left[\begin{array}{cccc}
1 & 0 & \cdots & 0 \\
1 & & & \\
0 & & A^{k, u} & \\
\vdots & & & \\
0 & & &
\end{array}\right]
$$

Then it can be shown by induction that

$$
\left(E^{k, u}\right)^{\alpha}=\left[\begin{array}{cccc}
1 & 0 & \cdots & 0 \\
S_{\alpha+u+k-1} & & & \\
S_{\alpha+u+k-2} & & \left(A^{k, u}\right)^{\alpha} & \\
\vdots & & & \\
S_{\alpha-1} & & &
\end{array}\right] .
$$

\section{References}

[1] Akuzum, Y., Hiller, J., \& Deveci, O. The Arrowhead-Pell Sequences, in press.

[2] Atanassov, K. T., Deford, D. R., \& Shannon, A. G. (2015) Pulsated Fibonacci recurrences. Fibonacci Quart., 52, 5, 22-27.

[3] Brualdi, R. A., \& Gibson, P. M. (1997) Convex polyhedra of doubly stochastic matrices I: Applications of permanent function, J. Combin. Theory, 22, 194-230.

[4] Budden, M., Hiller, J., \& Rapp, A. (2015) Generalized Ramsey theorems for $r$-uniform hypergraphs, Aust. J. Comb., 63, 1, 142-152.

[5] Chen, W. Y. C., Louck, J.C. (1996) The combinatorial power of the companion matrix, Linear Algebra Appl., 232, 261-278.

[6] Dawson, R., Gabor, G., Nowakowski, R., \& Wiens, D. (1985) Random Fibonacci-type sequences, Fibonacci Quart., 23, 169-176. 
[7] Deveci, O. (2016) The Pell-circulant sequences and their applications, Maejo Int. J. Sci. Technol., 10(3), 284-293.

[8] Deveci, O., \& Shannon, A. G. (2017) On The Adjacency-Type Sequences, Int. J. Adv. Math., $2017,2,10-24$.

[9] Gultekin, I., \& Deveci, O. (2016) On The Arrowhead-Fibonacci Numbers, Open Mathematics, 14, 1, 1104-1113.

[10] Hofstadter, D. R. (1979) Gödel, Escher, Bach: An eternal golden braid, Basic Books, NY.

[11] Kalman, D. (1982) Generalized Fibonacci numbers by matrix methods, Fibonacci Quart., $20,1,73-76$.

[12] Kilic, E. (2009) The generalized Pell $(p, i)$-numbers and their Binet formulas, combinatorial representations, sums, Chaos, Solitons Fractals, 40, 4, 2047-2063.

[13] Kilic, E., \& Tasci, D. (2006) The Generalized Binet Formula, Representation and Sums of The Generalized Order- $k$ Pell Numbers, Taiwan. J. Math., 10, 6, 1661-1670.

[14] Lancaster, P., \& Tismenetsky, M. (1985) The theory of matrices, Academic Press.

[15] Lidl, R., \& Niederreiter, H. (1994) Introduction to finite fields and their applications, Cambridge University Press.

[16] Shannon, A. G., \& Bernstein, L. (1973) The Jacobi-Perron Algorithm and the Algebra of Recursive Sequences, Bull. Australian Math. Soc., 8, 2, 261-277.

[17] Shannon, A. G., \& Horadam, A. F. (1994) Arrowhead curves in a tree of Pythagorean triples, Internat. J. Math. Ed. Sci.Technol., 25, 2, 255-261.

[18] Sloane, N. J. A. Sequences A000045/M0692, A000073/M1074, A000078/M1108, A001591, A001622, A046698, A058265, A086088, and A118745, The On-Line Encyclopedia of Integer Sequences.

[19] Stakhov, A. P., \& Rozin, B. (2006) Theory of Binet formulas for Fibonacci and Lucas p-numbers, Chaos Solitons Fractals, 27, 1162-1167.

[20] Tasci, D., \& Firengiz, M. C. (2010) Incomplete Fibonacci and Lucas p-numbers, Math. Comput. Modelling, 52, 1763-1770.

[21] Tasyurdu, Y., \& Deveci, O. (2017) The Fibonacci Polynomials in Rings, Ars Comb., 133, $355-366$.

[22] Tasyurdu, Y., \& Gultekin, I. (2013) On period of the sequence of Fibonacci polynomials modulo m, Discrete Dyn. Nat. Soc., 731482-1-731482-3.

[23] Tuglu, N., Kocer, E.G., \& Stakhov, A. P. (2011) Bivariate Fibonacci like p-polinomials, Appl. Math. Comput., 217, 24, 10239-10246. 